\title{
COVID-19 vaccination: Recommendations for management of patients with allergy or immune-based diseases
}

\author{
J Peter, $\mathrm{MB} \mathrm{ChB}, \mathrm{PhD}$ \\ Division of Allergy and Clinical Immunology, Department of Medicine, Faculty of Health Sciences, University of Cape Town, South Africa; \\ and Allergy and Immunology Unit, University of Cape Town Lung Institute, South Africa
}

Corresponding author: J Peter (jonny.peter@uct.ac.za)

\begin{abstract}
As South Africa continues to battle the second wave of SARS-CoV-2 infections, the imminent arrival of vaccines against COVID-19 offers hope. Vaccine roll-out has been accompanied by heightened media coverage that has created both excitement and anxiety, reporting on the shortened timeline of vaccine trials and approvals, as well as the recent series of anaphylaxis cases associated with the two approved mRNA COVID-19 vaccines. Patients with allergic and other immune-based diseases are subgroups especially concerned about vaccine safety and efficacy. This practice guideline offers broad recommendations for COVID-19 vaccination in various subgroups of allergic and immunebased disease, highlighting risk/benefit evaluation, and where and how routine vaccination should be altered.
\end{abstract}

S Afr Med J 2021;111(4):291-294. https://doi.org/10.7196/SAMJ.2021.v111i4.15576

The global pandemic of SARS-CoV-2 infection continues in the majority of the world's 195 countries, checked only by non-pharmaceutical interventions and restriction of human movement, and with catastrophic economic and social cost. The cost of human life, and the substantial morbidity in persons recovering from COVID19 , associated with attaining herd protection (estimated at $>60 \%$ of the population with immunity) through natural infection are unimaginable. ${ }^{[1]}$ Mass vaccination with effective and safe vaccines is therefore undoubtedly the best option for all countries, including South Africa (SA), to minimise the health, economic and social tragedies of this pandemic.

A vaccine is a biological product that can safely induce an immune response that confers protection against infection and/or disease on further exposure to a pathogen. Numerous vaccines are in widespread use, and arguably vaccines as a group are the biggest single public health success, with an estimated 2 - 3 million lives saved each year by current immunisation programmes. ${ }^{[2]}$ There are excellent reviews of basic concepts in vaccinology ${ }^{[3]}$ as well as an updated vaccine tracker detailing the pipeline and phase I - III clinical trial details of approved and late-phase COVID-19 vaccines (https://vac-lshtm.shinyapps. io/ncov_vaccine_landscape/\#); there are also now peer-reviewed publications of safety data for several of the candidate COVID-19 vaccines likely to be rolled out in SA, including the AstraZenecaOxford ChAdOx ${ }^{[4,5]}$ and Johnson \& Johnson Ad26 vaccines. ${ }^{[6]}$

The imminent approval and arrival of COVID-19 vaccines in SA has led to considerable focus on safe and rapid roll-out, and to individuals considering their own comorbidities and risk-benefits in receiving a specific approved vaccine. COVID-19 vaccines are the focus of heightened media coverage that has highlighted both the many vaccine candidates and the promise of vaccines, but also the shortened timeline of COVID-19 vaccine development, a series of cases of anaphylactic reactions to the two approved mRNA vaccines during roll-out in the UK and USA, ${ }^{[7,8]}$ and the pros and cons of lengthening the manufacturer's recommended dosing intervals for greater population coverage ${ }^{[9]}$ Add to this the backdrop of the overwhelming uncertainty surrounding a novel, deadly virus and a global rise in anti-vaccination sentiments, and it is unsurprising that media coverage has peaked anxiety about the safe use of SARS-CoV-2 vaccines in patients with allergic or immune-based diseases. In that context, this article aims to make broad recommendations to guide safe early uptake of COVID19 vaccination in patients with exisiting allergic or immune-based diseases. Table 1 provides the summary recommendations to guide vaccine centres, whether in outreach, primary, secondary or tertiary care facilities, where these patient groups may present for vaccination.

\section{Vaccine side-effects}

All medications, vaccines included, can have side-effects. The majority of vaccine side-effects are mild, and by-products of the early innate immune response. Common side-effects include injection site pain, redness and swelling, and systemic symptoms such as fever, headache and malaise; these occur in the first $1-2$ days following vaccination. ${ }^{[3]}$ Importantly for the population of patients with immunosuppression or immunodeficiencies, none of the current late-phase or approved candidate COVID-19 vaccines are live vaccines, as live vaccines such as the live intranasal influenza vaccine are contraindicated in patients with significant immunodeficiency. ${ }^{[10]}$ Serious side-effects from vaccines are rare and include allergy reactions and blood dyscrasias. ${ }^{[3]}$ Many people are alarmed by the identification of uncommon sideeffects to vaccines or medications after approval by regulatory bodies in the post-marketing surveillance period. However, this is to be expected. Regulatory approvals for all vaccines, irrespective of the timeline of development ( 1 year or 10 years), are given with safety studies completed in 3000 to tens of thousands of individuals. Rare side-effects may therefore not necessarily have been identified. ${ }^{[8]}$ Vaccine development is tightly controlled, and robust post-marketing surveillance systems are in place in SA and other countries to pick these side-effects up if they do occur. The SA National Department of Health will soon roll out an electronic reporting system for adverse events following immunisation (AEFI), allowing for national robust reporting of AEFI to occur. 


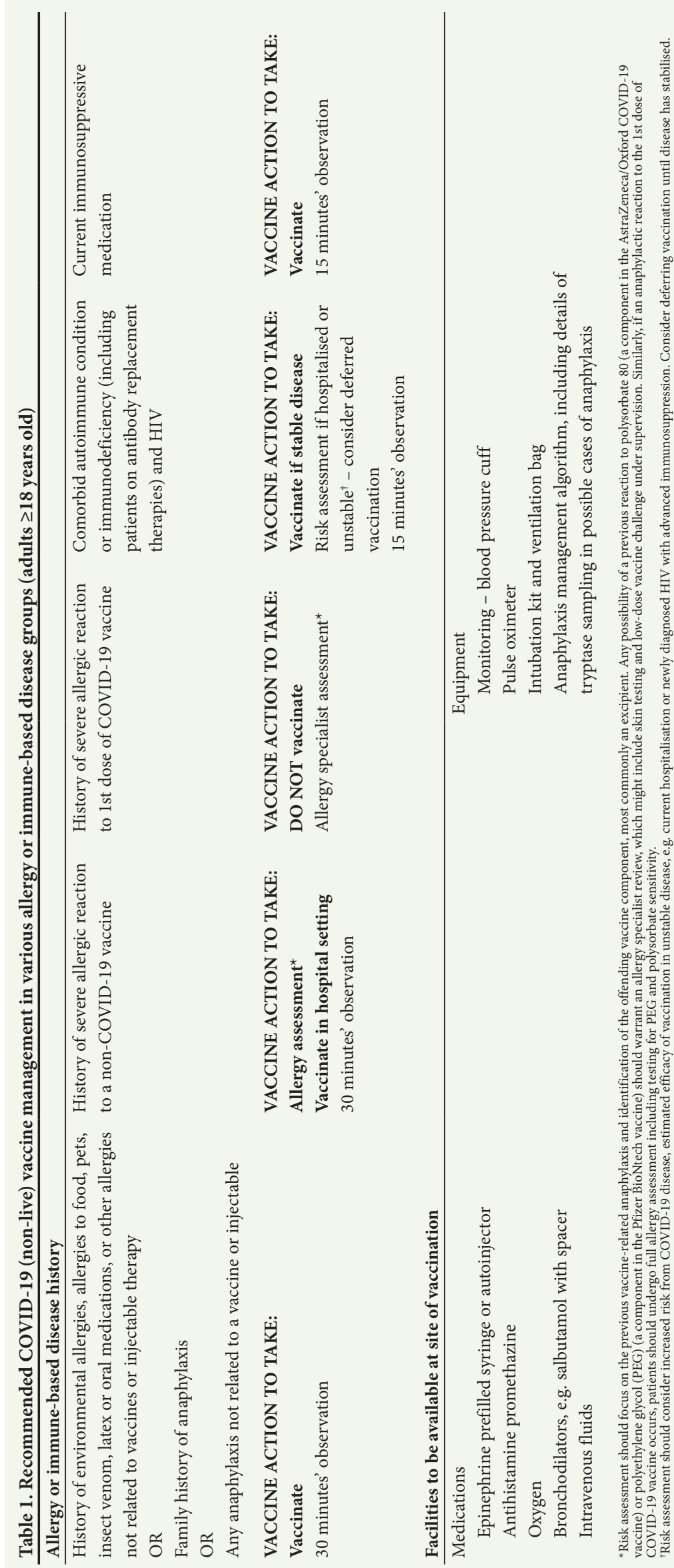

\section{Anaphylaxis to vaccines}

Anaphylaxis is a serious multisystem allergic reaction with a rapid onset; it can lead to death by asphyxiation, cardiovascular collapse and other complications. Fortunately, if promptly treated with intramuscular adrenaline, the majority of cases are quickly aborted, and studies of anaphylaxis fatality show the early administration of adrenaline to be a key predictor of survival. ${ }^{[1]}$ Anaphylaxis is a rare event following vaccination, at one case per million doses. ${ }^{[12,13]}$ Specific to the roll-out of the two mRNA COVID-19 vaccines, following an estimated 6 million doses and climbing, the prevalence of anaphylaxis appears to be estimated at between one case in 100000 400000 doses. $^{[7.8]}$ Anaphylactic reactions have been reported to several current vaccines, ${ }^{[12]}$ and the mechanisms of vaccine-associated anaphylaxis are incompletely understood, with both $\operatorname{IgE}$ and non-IgE mechanisms likely to be involved, depending on the offending agent. The inciting allergens can include vaccine antigen, residual non-human proteins, e.g. gelatin, or preservatives and stabilisers used in the vaccine formulation, known collectively as excipients. ${ }^{[8]}$ Excipients are usually published in the package inserts of licensed vaccines, and a useful summary table for the new COVID-19 vaccines under emergency use authorisation by the FDA or in late-phase study can be found in an article by Castells and Phillips. ${ }^{[8]}$ The likely inciting allergen in the two mRNA COVID-19 vaccines developed by PfizerBioNtech and Moderna is the polyethylene glycol (PEG) lipid conjugate that helps to provide a hydrophilic layer to prolong the half-life..$^{[8,14]}$

\section{Patients with allergy and immune-based diseases: 'Should I be vaccinated?'}

\section{General consideration of risks/benefits} of COVID-19 vaccination

Personal perception of risk is influenced by a complex intersection of past experiences, cultural values, emotional state, current paradigms of thinking, and the necessity to undertake actions involving increased risk. For instance, many people fear flying, yet have no problem driving long-distance in a motor vehicle; statistically, the risk of death is more than a 100 -fold higher from a motor vehicle accident compared with an airplane crash. Evidence-based medical treatment and strict guideline development processes have been established in healthcare to avoid individual biases in decision-making. The many uncertainties and media deluge around SARS-CoV-2 mean that individual perceptions of vaccination will vary substantially. Where possible, as healthcare providers we must: $(i)$ do our best to help individualise risks of severe COVID-19 disease and vaccination; (ii) be transparent about current evidence and where vaccine use would be offlabel; and (iii) respect individual autonomy while 
being aware of the utilitarian societal benefit of wide-scale vaccine uptake for herd protection.

The age-standardised risk of COVID-19 death in SA was reported at around 1 in 100000 population; ${ }^{[15]}$ however, this risk increases substantially with each decade, and with risk factors for severity including obesity, diabetes, hypertension and chronic organ diseases. ${ }^{[16]}$ There have been conflicting data on whether asthma, ${ }^{[17]}$ autoimmune diseases ${ }^{[18]}$ and immunodeficiency states, e.g. $H I V,{ }^{[16]}$ having had a transplant, ${ }^{[19,20]}$ immunosuppressive therapies ${ }^{[21]}$ or primary immunodeficiencies, ${ }^{[22-24]}$ significantly increase risk in addition to age and the aforementioned risk factors, but the majority of emerging data mostly indicate a risk of increased severity associated with these diseases. Data are still lacking for many subgroups of uncommon immune-based diseases. For the majority of individuals with allergic and immune-based disease comorbidities, the risk of severe COVID-19-related disease will outweigh safety concerns of vaccination.

Detailed efficacy data for a specific COVID-19 vaccine in the majority of immune-based disease subgroups, including HIV, will be unavailable at the time of emergency-use authorisation and roll-out, because groups with severe unstable disease or known immunosuppression are mostly excluded from the initial large phase III trials (see https://clinicaltrials.gov/ct2/show/NCT04444674 for example protocol from the ChAdOx1 nCov-19 trial). Fortunately, some stable HIV patients on antiretrovirals with suppressed viral loads have been included in clinical trials of most vaccines, and efficacy data will emerge in the coming months. The fact that there are no live COVID-19 vaccine candidates in the pipeline means that there will not be a contraindication to use in patients with secondary or primary immunodeficiency states or those receiving immunosuppressive therapies; however, these patients will need to be informed that use will be off-label and the efficacy of vaccination is unknown. It is hoped that the collection of large-scale roll-out data will provide this information in time, but extra counselling should be provided to these off-label patient groups to encourage ongoing rigorous use of non-pharmaceutical interventions to reduce the likelihood of infection.

Table 1 summarises pragmatic COVID-19 vaccine recommendations for different subgroups of patients with allergic or immunebased disease.

\section{Known allergic diseases}

Atopic diseases, including allergic rhinitis, asthma, atopic dermatitis and food or drug allergy, affect up to $30 \%$ of the SA population. ${ }^{[25]}$ Although vaccine- or drug-induced anaphylaxis is more common in atopic individuals, the risk is very small, and importantly, reactions are allergen-specific, meaning that unless the offending agent is a cross-reacting substance such as an excipient, that may be found in more than one vaccine or drug, even prior drug- or vaccine-induced anaphylaxis may not be an absolute contraindication to receiving a COVID-19 vaccine. Two important practice points should improve safety: (i) patients with prior anaphylaxis or severe allergic disease should be observed for 30 rather than 15 minutes after vaccination, as the majority of drug- or vaccine-induced anaphylaxis occurs within the first 30 minutes following the dose; and (ii) individuals with prior vaccine-associated anaphylaxis should have an allergy assessment and review by a specialist to ensure safety, and particularly the absence of sensitivity to excipients, e.g. polysorbate 80 or PEG in COVID-19 vaccines. A detailed review of allergy specialist assessment in the context of suspected reactions to PEG, polysorbate or the two recently approved mRNA COVID-19 vaccines is available for reference. ${ }^{[26]}$ It is recommended that vaccination should be done in facilities fully equipped and with experienced staff to manage allergic reactions should they arise. ${ }^{[11]}$

\section{Known autoimmune disease}

Autoimmune or inflammatory disease as a group is common, and severe disease requires immunosuppression, including smallmolecule therapies and biologicals. Vaccines stimulate an immune response and may contain adjuvants, raising mostly theoretical concerns around exacerbating inflammatory disease. To date, no studies have substantiated these with a robust evidence base. Furthermore, immune dysregulation and its treatment can often result in increased susceptibility to infections, and booster vaccination is frequently recommended, as for pneumococcus and influenza pathogens. ${ }^{[27]}$ Although limited data are available, patients with autoimmune disease and frequently with end-organ damage are likely to be at increased risk of severe COVID-19 disease and complications. ${ }^{[18]}$ COVID-19 vaccination is therefore preferable to non-vaccination and should be encouraged.

\section{Known immunodeficiency (primary or secondary) and immunosuppressive therapy}

There are several groups of patients, largely excluded from current phase III vaccine trials, with immunodeficiency, including patients with: (i) inborn errors of immunity; (ii) malignancies, especially haematological cancers with immunoparesis; and (iii) haematopoietic stem cell and solid organ transplant patients, who are all considered at increased risk for severe COVID-19 disease. ${ }^{[19,20,22-24]}$ The immunological response to COVID-19 vaccination is likely to be heterogeneous among these patients. HIV patients are another very large and important group of immunodeficiency patients in SA, but fortunately relevant trials have included stable HIV patients on antiretrovirals and with suppressed viral loads, so specific immunogenicity and vaccine efficacy data will soon be available to guide use in this large and important SA patient group. Lastly, patients on chronic immunosuppressive therapy are another at-risk group where vaccination will be off-label. Fortunately, however, safety of non-live vaccines is not likely to be an issue or a contraindication to use for any of these groups, and where possible it will be reasonable to consider off-label vaccination. Individual patients should contact their specialist if they are concerned, for a case-by-case risk assessment. All patients in these groups should be clearly counselled that the effectiveness of vaccination will be unknown and every effort to avoid infection should still be implemented, especially during surges.

\section{Additional safety precautions}

Places of vaccination should all be equipped to manage cases of anaphylaxis should they occur. Preparation includes the availability of emergency equipment, the latest anaphylaxis algorithm, and staff trained in the management of anaphylaxis. It is recommended that prior to the start of each vaccination day, the staff team should identify roles and responsibilities in the case of an emergency, including the appropriate evacuation of patients to intensive care settings. High-risk allergic patients should not be vaccinated by small mobile outreach vaccine teams, where availability of equipment and trained staff is limited. The majority of drug- and vaccine-induced anaphylaxis occurs within 30 minutes of dosing, including the recent cases related to the mRNA COVID-19 vaccines, with $71 \%$ of allergic reactions to the Pfizer BioNTech COVID-19 mRNA vaccine occurring within 15 minutes of vaccination. ${ }^{[20]}$ It is therefore recommended that allergic patients should be observed at the vaccine health centre for 30 rather than the routine 15 minutes after dosing. 


\section{Conclusions}

Vaccination offers the opportunity for both individual and, with sufficient coverage, herd protection against SARS-CoV-2 infection and disease; if vaccination is safe and available, it should therefore be done. Vaccination should only be contraindicated in the very small group of patients with a prior anaphylactic reaction to either the first dose of COVID-19 vaccine or an ingredient in the vaccine formulation, e.g. PEG. However, given the emerging nature of efficacy data and the exclusion of certain subgroups of patients with immunebased disease, counselling of patients must make it clear regarding where vaccine effectiveness is unknown and may be reduced, and all other efforts to avoid infection must continue.

\section{Declaration. None.}

Acknowledgements. Editorial inputs from Elizabeth Phillips.

Author contributions. Sole author.

Funding. None.

Conflicts of interest. None.

1. Buss LF, Prete CA Jr, Abrahim CMM, et al. Three-quarters attack rate of SARS-CoV-2 in the Brazilian Amazon during a largely unmitigated epidemic. Science 2021;371(6526):288-292. https://doi org/10.1126/science.abe 9728

2. World Health Organization. Global Vaccine Action Plan 2011 - 2020. Geneva: WHO, 2020. https:// World Health Organization. Global Vaccine Action Plan 2011 - 2020. Geneva: WHO, 2020. https://
www.who.int/teams/immunization-vaccines-and-biologicals/strategies/global-vaccine-action-plan www.who.int/teams/immu

3. Pollard AJ, Bijker EM. A guide to vaccinology: From basic principles to new developments. Nat Rev Immunol 2020 (epub 22 December 2020). https://doi.org/10.1038/s41577-020-00479-7

4. Ramasamy MN, Minassian AM, Ewer KJ, et al. Safety and immunogenicity of ChAdOx1 nCoV-19 vaccine administered in a prime-boost regimen in young and old adults (COV002): A single-blind, randomised, controlled, phase 2/3 trial. Lancet 2021;396(10267):1979-1993. https://doi.org/10.1016/ s0140-6736(20)32466-1

5. Voysey M, Clemens SAC, Madhi SA, et al. Safety and efficacy of the ChAdOxl nCoV-19 vaccine (AZD1222) against SARS-CoV-2: An interim analysis of four randomised controlled trials in Brazil, South Africa, and the UK. Lancet 2021;397(10269):99-111. https://doi.org/10.1016/s01406736(20)32661-1

6. Sadoff J, Le Gars M, Shukarev G, et al. Interim results of a phase 1-2a trial of Ad26.COV2.S Covid-19 vaccine. N Engl J Med 2021 (epub 13 January 2021). https://doi.org/10.1056/NEJMoa2034201

7. Allergic reactions including anaphylaxis after receipt of the first dose of Pfizer-BioNTech COVID-19

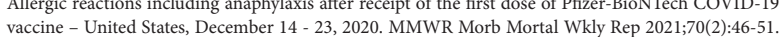
vaccine - United States, December $14-23,202$

8. Castells MC, Phillips EJ. Maintaining safety with SARS-CoV-2 vaccines. N Engl J Med 2020 (epub 30 December 2020). https://doi.org/10.1056/NEJMra2035343

9. News Analysis. COVID-19 vaccination: What's the evidence for extending the dosing interval? BMJ 2021;372:n18. https://doi.org/10.1136/bmj.n18
10. Eibl MM, Wolf HM. Vaccination in patients with primary immune deficiency, secondary immune deficiency and autoimmunity with immune regulatory abnormalities. Immunotherapy immune deficiency and autoimmunity with immune

11. Lieberman P, Nicklas RA, Randolph C, et al. Anaphylaxis - a practice parameter update 2015. Ann Allergy Asthma Immunol 2015;115(5):341-384. https://doi.org/10.1016/j.anai.2015.07.019

12. McNeil MM. Vaccine-associated anaphylaxis. Curr Treat Options Allergy 2019;6(3):297-308. https:// doi.org/10.1007/s40521-019-00215-0

13. McNeil MM, Weintraub ES, Duffy J, et al. Risk of anaphylaxis after vaccination in children and adults. J Allergy Clin Immunol 2016;137(3):868-878. https://doi.org/10.1016/j.jaci.2015.07.048

14. Caballero ML, Quirce S. Excipients as potential agents of anaphylaxis in vaccines: Analyzing the formulations of the current authorized COVID-19 vaccines. J Investig Allergol Clin Immunol 2021;31(1). https://doi.org/10.18176/jiaci.0667

15. Pillay-van Wyk V, Bradshaw D, Groenewald P, et al. COVID deaths in South Africa: 99 days since South Africa’s first death. S Afr Med J 2020;110(11):1093-1099. https://doi.org/10.7196/SAMJ.2020. v110i11.15249

16. Boulle A, Davies MA, Hussey H, et al. Risk factors for COVID-19 death in a population cohort study from the Western Cape Province, South Africa. Clin Infect Dis 2020;ciaal198 (epub 29 August 2020). https://doi.org/10.1093/cid/ciaal198

17. Terry PD, Heidel RE, Dhand R. Asthma in adult patients with COVID-19: Prevalence and risk of severe disease. Am J Respir Crit Care Med 2021 (epub 25 January 2021). https://doi.org/10.1164/ rccm.202008-3266OC

18. Liu Y, Sawalha AH, Lu Q. COVID-19 and autoimmune diseases. Curr Opin Rheumatol 2020;33(2):155162. https://doi.org/10.1097/bor.0000000000000776

19. Raja MA, Mendoza MA, Villavicencio A, et al. COVID-19 in solid organ transplant recipients: A systematic review and meta-analysis of current literature. Transplant Rev (Orlando) 2020;35(1):100588. https://doi.org/10.1016/j.trre.2020.100588

20. Sharma A, Bhatt NS, St Martin A, et al. Clinical characteristics and outcomes of COVID-19 in haematopoietic stem-cell transplantation recipients: An observational cohort study. Lancet Haematol 2021 (epub 19 January 2021). https://doi.org/10.1016/s2352-3026(20)30429-4

21. Andersen KM, Mehta HB, Palamuttam N, et al. Association between chronic use of immunosuppressive drugs and clinical outcomes from coronavirus disease 2019 (COVID-19) hospitalization: A retrospective cohort study in a large US health system. Clin Infect Dis 2021 (epub 7 January 2021). A retrospective cohort study in a large

22. Shields AM, Burns SO, Savic S, et al. COVID-19 in patients with primary and secondary immunodeficiency: The United Kingdom experience. J Allergy Clin Immunol 2020 (epub 14 December 2020). https://doi.org/10.1016/j.jaci.2020.12.620

23. Meyts I, Bucciol G, Quinti I, et al. Coronavirus disease 2019 in patients with inborn errors of immunity: An international study. J Allergy Clin Immunol 2020 (epub 24 September 2020). https:// doi.org/10.1016/j.jaci.2020.09.010

24. Delavari S, Abolhassani $\mathrm{H}$, Abolnezhadian F, et al. Impact of SARS-CoV-2 pandemic on patients with primary immunodeficiency. J Clin Immunol 2020 (epub 1 December 2020). https://doi.org/10.1007/ s10875-020-00928-x

25. Bousquet JJ, Schünemann HJ, Togias A, et al. Next-generation ARIA care pathways for rhinitis and asthma: A model for multimorbid chronic diseases. Clin Transl Allergy 2019;9:44. https://doi. org/10.1186/s13601-019-0279-2

26. Banerji A, Wickner P, Saff R, et al. mRNA vaccines to prevent COVID-19 disease and reported allergic reactions: Current evidence and suggested approach. J Allergy Clin Immunol Pract 2020 (epub 30 December 2020). https://doi.org/10.1016/j.jaip.2020.12.047

27. Ben Nessib D, Fazaa A, Miladi S, et al. Do immunosuppressive agents hamper the vaccination response in patients with rheumatic diseases? A review of the literature. Therapie 2020 (epub 3 September 2020). https://doi.org/10.1016/.j.therap.2020.08.002

Accepted 27 January 2021 